Dewing, S., et al.. (2011). The Feasibility of implementing a sexual risk reduction intervention in routine clinical practice at an ARV clinic in Cape Town: a case study. AIDS BEHAVIOUR, 15(5):905-10.

\title{
The Feasibility of Implementing a Sexual Risk Reduction Intervention in Routine Clinical Practice at an ARV Clinic in Cape Town: A Case Study
}

Sarah Dewing • Catherine Mathews •Nikki Schaay • Allanise Cloete • Leickness Simbayi $\bullet$ Mickey Chopra

\begin{abstract}
This case study with one lay adherence counsellor assessed the implementation of Options for Health, a sexual risk-reduction intervention based on Motivational Interviewing (MI), in an antiretroviral clinic in Cape Town, South Africa. In most cases Options was not delivered with fidelity and less than one-third of intended recipients received it; the counsellor often forgot to do Options, was unsure how to deal with particular cases and felt that there was not always time to do Options. Options was not implemented in a way that was consistent with MI. Revisions to the implementation plan and training programme are required.
\end{abstract}

Keywords Positive prevention • Motivational interviewing • Lay counsellors • Feasibility

S. Dewing (\&) • C. Mathews • M. Chopra Health Systems Research Unit, Medical Research Council, P.O. Box 19070, Tygerberg, Cape Town 7505, South Africa e-mail: sarah.dewing@mrc.ac.za

C. Mathews School of Public Health and Family Medicine, University of Cape Town, Cape Town, South Africa

N. Schaay School of Public Health, University of the Western Cape, Cape Town, South Africa

A. Cloete $\bullet$ L. Simbayi Social Aspects of HIV/AIDS and Health, Human Sciences Research Council, Cape Town, South Africa

\section{Introduction}

In 2008 an estimated 400,000 HIV positive South Africans were receiving antiretroviral (ARV) treatment [1], including over 23,000 adults in the Cape Town metropolitan area [2]. A recent study revealed that, of HIV positive people initiating ARV treatment in public health clinics in Cape Town, 44.7\% had had unprotected sex at last sex [3]. Given that ARVs do not eliminate the possibility of HIV transmission, there is concern that unprotected sex within this population has implications not only for the spread of HIV, but for the spread of drug resistant strains as well [4]. 
Because of the repeated interaction occurring between health workers and patients within HIV clinical care, this setting offers the opportunity for the provision of ongoing risk-reduction activities among people living with HIV (PLHIV) [5]. In collaboration with the Western Cape Provincial and City Departments of Health, we conducted this study in preparation for the implementation of a sexual-risk reduction intervention called Options for Health using lay ARV adherence counsellors in ARV clinics across the Cape Town metropolitan area.

\section{The Options for Health Intervention}

Options for Health is a sexual risk reduction intervention based on the Information, Motivation and Behavioural (IMB) Skills model of behaviour change, using Motivational Interviewing (MI) techniques to deliver HIV risk reduction information, motivation and behavioural skills content [5]. The Options counselling protocol consists of eight steps in which the provider (1) introduces the discussion of safer sex, (2) assesses the patient's risk behaviours, (3) determines how important it is to the patient to change their risk behaviour, (4) determines how confident the patient is that they can change their risk behaviour, (5) elicits information-, motivation-, and behavioural skills- based strategies from the patient for overcoming barriers and moving towards (or maintaining) change, (6) negotiates an individually-tailored risk reduction behaviour change goal or plan of action, (7) documents details of the counselling session on a standardized patient record form for use in follow-up counselling sessions and (8) documents the agreed upon plan on the Options action plan form [5]. The intervention has been designed for use in high patient load clinic settings and initial Options sessions should take no more than $15 \mathrm{~min}$.

\section{Background}

Increasing service needs and shortages of professional health workers in South Africa has led to the promotion of 'task shifting' (that is the delegation of responsibilities to the "lowest" category that can successfully perform them) as one way in which to expand capacity for service delivery [6]. Lay health workers are individuals carrying out functions related to health care delivery but having no formal professional or tertiary education [7]. South Africa's large- scale ARV treatment programme relies heavily on lay health workers to prepare patients for treatment and for providing adherence support [8]. Originally developed in the United States, Options for Health has been adapted and piloted in a randomized control trial in an urban HIV care clinic in Kwa-Zulu Natal (KZN), South Africa [5]. Three of five lay ARV adherence counsellors working at the clinic were randomly assigned to receive training in the Options counselling protocol over a period of 3 days, and a social worker who was a member of the clinic staff provided weekly supervision. Participants were randomly assigned to a counsellor at study enrolment. Among the three Options-trained counsellors, the intervention was delivered in $99 \%$ of routine patient visits with fidelity (counsellors implemented 7 of the 8 protocol steps in $78.8 \%$ of Options for Health counselling sessions). Patients having received the intervention reported a significant decrease in unprotected sexual events over a 6 month period, as compared to patients in the standard-of-care control group. However two recent studies conducted in the

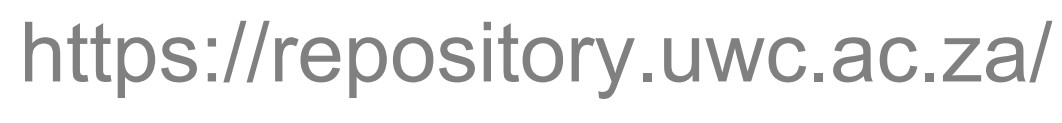


Western Cape question the ability of lay counsellors to implement counselling techniques associated with MI. One study in which 38 lay counsellors were trained over 3 days in a counselling approach aligned with the key principles of MI found that counsellors did not achieve beginning proficiency in the counselling technique (as assessed by the Motivational Interviewing and Treatment Integrity Code (MITI)) [9]. Similarly, lay counsellors trained by Evangeli et al. [10] were found to improve in some skills associated with MI but very few achieved a level of beginning proficiency when evaluated using the same tool.

While the efficacy of Options for Health has been demonstrated in one South African ARV clinic, the feasibility of incorporating the intervention into routine adherence counselling practice remains unclear. This case study represents the first step in moving this evidence-based intervention into practice, and was conducted in order to investigate the impact of incorporating Options into adherence counselling on the running of the clinic, to monitor implementation in terms of fidelity to the counselling protocol, the coverage of the intervention and the quality of intervention delivery.

\section{Methods}

\section{Setting}

Options was implemented from July to October 2008 in an ARV clinic of high patient load (averaging 1,128 patients per month over first 6 months of 2008) [2]. ARV clinic staff consisted of two adherence counsellors, two nursing sisters, two (and sometimes three) doctors and five patient advocates (PA's). ${ }^{1}$ Upon arrival at the clinic, patients have their pills counted by either a nurse or a PA and are referred for adherence counselling if they are found to have been non-adherent since their last visit. These patients are referred to as ARV 'follow-up' patients.

\section{Procedure}

One of the two adherence counsellors employed at the clinic participated in a 4-day Options master training course that was held in January 2008. This counsellor was selected for participation with the master trainers by the counselling coordinator at the NGO with which he is employed because she felt he was dedicated, enthusiastic and a 'good' counsellor. In terms of education he had completed Grade 12 and had 6 years of counselling experience in the field of HIV/AIDS. The Options training programme included modules on the intervention protocol, sex and drug risk for HIV and disclosure. Implementation began 4 months following training. The counsellor took part in a $2 \mathrm{~h}$ "booster" training session with one of the master trainers on the day before he began to implement the intervention with his patients. The counsellor was asked to use the Options counselling protocol to address sexual risk behaviour with all ARV 'follow-up' patients referred to him for counselling.

\footnotetext{
1 "Patient Advocates" are community workers who provide support to patients by conducting home visits prior to treatment initiation and also between their clinical appointments to provide advice on adherence and healthy living.
} 
To assess the impact of the intervention on the day-today running of the clinic we conducted observations of the time the Options-trained adherence counsellor spent with each patient receiving counselling over six typical days, and informal conversations were conducted with the two ARV nurses, one doctor and three of the PAs during the first month of the study. Fidelity to the counselling protocol was assessed by review of the Options patient record forms that are completed as a part of the 8 step counselling protocol; the record forms require detail corresponding to 7 of the 8 steps to be filled in at the end of each session. Intervention coverage was assessed by comparing the number of patient record forms completed with the number of ARV follow-up patients seen for counselling as routinely recorded in the counsellors' patient diary. The quality of the delivery of the intervention was assessed by review of the content of the patient record forms and by evaluating the counsellors' use of MI consistent counselling skills. An Options counselling session was audio- recorded, transcribed and translated from Xhosa into English by the Options fieldworker. The transcript was evaluated by two independent raters using the MITI 3.0

[11] . A 5-point Likert scale is used to rate practitioners on 3 dimensions (Evocation, Collaboration, Autonomy/support) while behaviour counts are used to tally instances of particular behaviours (e.g. open vs. closed questions; simple vs. complex reflections). Because of concern regarding the appropriateness of using the MITI to evaluate lay counsellors' ability to utilize MI skills, the researchers evaluated the same transcript using the Behaviour Change Counselling Index (BECCI) for comparison. Lane et al.

\section{[12]}

[13] describe adaptations of MI designed for brief healthcare consultations and omitting some of the more psy- chotherapeutic elements of MI as 'behaviour change counselling', and developed the BECCI to assess skills associated with such counselling. The BECCI categorizes 11 aspects of practitioner skill and the degree to which each was carried out is rated on a 5-point Likert scale. The 11 items focus on the occurrence of behaviour-change talk as well as a few micro-skills (questions, empathic listening statements and summaries).

\section{Results}

The Impact of Options on the Routine Running of the Clinic

ARV clinic staff were supportive of the idea of a sexual risk reduction intervention and were keen to see a reduction in sexual risk behaviour among their patients. The counsellor was enthusiastic about the intervention ("in terms of counselling it (has) helped me a lot to be able to deal with clients") and particularly liked the structure that the eight steps of the Options protocol lent to counselling sessions. During our period of observation the time

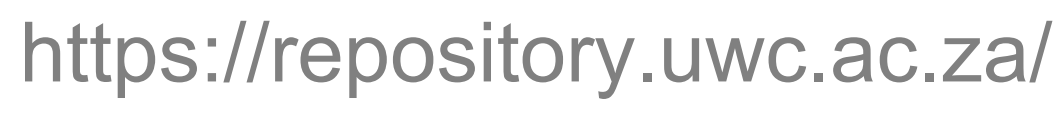


spent with patients who did not receive Options $(n=6)$ averaged $11 \mathrm{~min}$, while the time spent with patients who did receive Options $(n=28)$ averaged $18 \mathrm{~min}$. While there was an increase in the amount of time the counsellor spent with patients receiving Options, this did not negatively impact the work of the ARV nurses, doctors or the PAs; in fact many reported that they had forgotten that we were conducting a study at the clinic.

\section{Intervention Fidelity}

A review of the patient record forms showed that the adherence counsellor implemented 7 of the 8 protocol steps in $36 \%$ of Options counselling sessions. In $17 \%$ of sessions 6 steps were implemented and in $35 \%$ of sessions five steps were implemented. In the majority of sessions in which six steps were completed a copy of the action plan had not been handed to the patient; the counsellor reported that patients did not like to take the action plans he wrote up. In the majority of sessions in which only five steps were implemented no action plan had been negotiated, and it was clear from the record forms that these cases were ones in which the client had reported consistently practicing protected sex or no current sexual activity. In the remaining $12 \%$ of sessions four or fewer steps had been completed. The counsellor reported that he had not known how to handle the issues that arose in these particular cases and had therefore not completed these counselling sessions. One case was one in which the client had expressed an inability to ejaculate while wearing male condoms, and another was one in which a male client had spoken of his male sexual partners. According to the counsellor, this was the first time he had been confronted by homosexuality in his work, and he had not known how to respond to the patient.

\section{Intervention Coverage}

A comparison of the number of patient record forms collected from the counsellor with the number of ARV follow- up patients having received counselling revealed that $88 \%$ of intended recipients received Options during the first month of implementation while only $8 \%$ of intended recipients received Options during the last month of implementation. In total, only $31 \%$ of the intended recipients received Options over the 15 weeks of implementation. Reasons given by the counsellor for not having conducted Options counselling sessions with patients who should have received them were that he forgot to do

Options with some patients and he did not know how to deal with the issues that arose in particular sessions and thus did not complete these sessions using the Options protocol. The counsellor also did not feel that he had the time to do Options with each patient; in addition to the fact that Options had to be done after (and in addition to) the standard-of-care adherence counselling, he reported that patients' stories would often change as they progressed through the session. In such cases he would then need to go back a few steps in order to try to understand and deal with the 'real' issue, and this affected the amount of time spent with the patient. Lastly, the completion of an Options patient record form for each patient was perceived as an additional burden on his time and workload.

\section{Quality of Intervention Delivery}

The MITI results are presented in Table 1 below where it can be seen that the counsellors' scores fall below the competency threshold provided by the tool for each criteria. In addition

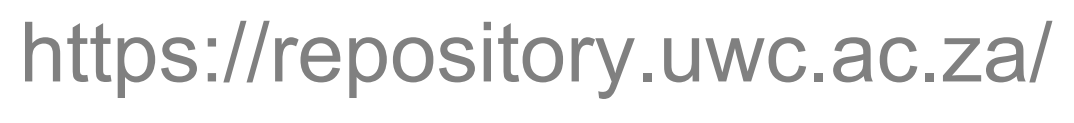


the BECCI scores reveal that the counselling session was not conducted in a way that was consistent with behaviour change counselling.

A review of the patient record forms revealed that the counsellor was not always able to implement the Options counselling protocol in such a way that it seemed likely that patients could attempt to reduce their sexual risk behaviour with the possibility of success. Many of the action plans appeared to the researchers to be unrealistic and unachievable. For example, one patient reported that he did not use condoms when he was drunk; the plan of action negotiated in response was "to stop drinking and use condoms every time he has sex". Other seemingly superficial strategies included: "not having intercourse when there are no condoms", "try not use alcohol and refuse if friends are offering" and, for some clients who were not currently sexually active, "disclosure to new (sexual) partners". Strategies that appeared to be more achievable usually involved small steps towards the desired behaviour such as discussing the importance of safer sex with sexual partners who are aware of their partners' HIV status but reluctant to use condoms, and keeping condoms closer to the bed so that they are easily accessible when needed.

\begin{tabular}{|l|l|l|l|l|}
\hline Criteria & Rater 1 & Rater 2 & $\begin{array}{l}\text { Competency } \\
\text { threshold }\end{array}$ & $\begin{array}{l}\text { Proficiency } \\
\text { threshold }\end{array}$ \\
\hline Global spirit rating & 2 & 2 & 3.5 & 4 \\
\hline Percent complex reflections (\%) & 0 & 0 & 40 & 50 \\
\hline Percent open questions (\%) & 38 & 32 & 50 & 70 \\
\hline Reflection to question ratio $^{\text {a }}$ & 0.32 & 0.32 & 1 & 2 \\
\hline Percent MI adherent (\%) (35 & 50 & 90 & 100 \\
\hline
\end{tabular}

\begin{tabular}{|c|c|c|c|}
\hline \multicolumn{4}{|l|}{$\begin{array}{l}\text { The Behaviour Change Counselling Index } \\
\text { (BECCI) }\end{array}$} \\
\hline Item & Rater 1 & Rater 2 & Scoring system \\
\hline Global score $^{b}$ & 0.45 & 0.45 & $\begin{array}{l}0=\text { Not at all } \\
1=\text { Minimally } \\
2=\text { To some extent } \\
3=\text { A good deal } \\
4=\text { A great extent }\end{array}$ \\
\hline \multicolumn{4}{|l|}{$\begin{array}{l}\text { Examples of the BECCI Items and the } \\
\text { counsellors' scores }\end{array}$} \\
\hline $\begin{array}{l}\text { Practitioner invites patient to talk about } \\
\text { behaviour change }\end{array}$ & 0 & 0 & $\begin{array}{l}\text { Please refer to scoring system above for the } \\
\text { interpretation } \\
\text { of this score }\end{array}$ \\
\hline $\begin{array}{l}\text { Practitioner acknowledges challenges about } \\
\text { behaviour } \\
\text { change that patient faces }\end{array}$ & 1 & 1 & $\begin{array}{l}\text { Please refer to scoring system above for the } \\
\text { interpretation } \\
\text { of this score }\end{array}$ \\
\hline $\begin{array}{l}\text { When practitioner provides information it is } \\
\text { sensitive to patient } \\
\text { concerns and understanding }\end{array}$ & 1 & 0 & $\begin{array}{l}\text { Please refer to scoring system above for the } \\
\text { interpretation } \\
\text { of this score }\end{array}$ \\
\hline $\begin{array}{l}\text { Practitioner and patient exchange ideas about } \\
\text { how the patient } \\
\text { could change current behaviour }\end{array}$ & 1 & 1 & $\begin{array}{l}\text { Please refer to scoring system above for the } \\
\text { interpretation } \\
\text { of this score }\end{array}$ \\
\hline
\end{tabular}

a The ratio of the number of reflective listening statements made to the number of open and closed questions asked

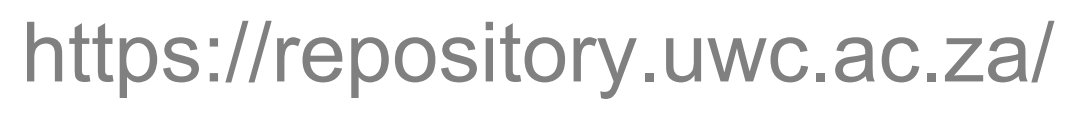


$\mathrm{b}$ Scores for the 11 items of the BECCI are averaged to produce a global score corresponding to the points given on the Likert scale. The full BECCI scale is available at http://www.motivationalinterview.org/library/BECCIForm.pdf

\section{Discussion}

This research identified a number of potential barriers to the provision of ongoing HIV riskreduction counselling as a part of standard-of-care in low-resource, primary care ARV facilities. A limitation associated with using ARV adherence counsellors to deliver Options is that only non- adherent patients are referred for counselling, leaving many people who may benefit from the intervention unexposed. Also, because referrals for counselling are generally made on the basis of pill counts conducted at each clinic visit, it is difficult to ensure that people return (or are referred) for follow-up counselling sessions.

The inclusion of Options into routine adherence counselling practice was shown to increase the amount of time spent with patients and this was one of the main reasons why patients who were referred for counselling did not receive the intervention (the counsellor often chose to deliver standard-of-care counselling instead). A solution may be to include ARV non-adherence as a focus of the intervention in the next phase of the Options for Health: Western Cape project, and to tailor delivery so that it is more consistent with current practice. For example, counsellors currently address the issue of safer sexual practice in one of three treatment 'work-up' sessions conducted with patients who are waiting to initiate ART. Counsellors could use Options to focus on sexual risk behaviour in these sessions while using Options to focus on non- adherence in 'follow-up' sessions. In this way Options would fit more discretely into adherence counselling practice and the probability of it being 'forgotten' or ignored due to lack of time and other competing priorities (like ARV adherence) should be reduced.

In contrast to the fidelity with which Options was delivered in the KZN pilot study, 5 or fewer of the 8 steps of the Options protocol were delivered in almost half (47\%) of all Options sessions conducted. In addition it appeared (from the perspective of the researchers) that the counsellor found it difficult to negotiate goals for behaviour change with patients that were likely to be achievable, and did not know how to use the 8 step protocol to encourage the maintenance or future practice of safer sexual behaviour if patients reported no current or unprotected sexual activity. While a limitation of this study was the delay between training and implementation, these findings point to the importance of providing supervision and the opportunity for 'up-skilling' and trouble-shooting difficult cases; a key difference in implementation between the two studies was the provision of weekly on-site supervision by a staff member (and social worker) in KZN. Supervision of this intensity is not available to lay counsellors working in the Western Cape, but Options-specific support could be included in the monthly mentoring meetings that are run by psychologists/social workers and provided by their NGOs. 
The KZN study did not assess the degree to which the counsellors' were using counselling techniques associated with MI when delivering Options; according to the MITI and the BECCI, the counsellor in this study was not using the 8 step protocol in a way that was consistent with behaviour change counselling or its' parent method, MI. Although this assessment was made based on the work of one counsellor in one transcript this finding is consistent with those of Mash et al. [9] and Evangeli et al. [10]. While this observation raises the question as to what extent an Options session needs to exhibit the characteristics of MI in order to result in behaviour change, monitoring the quality of the delivery of Options in terms of counselling technique will nevertheless be an important part of assessing whether the intervention is being delivered as intended when rolled out to facilities across the Cape Town area, and in evaluating the ability of lay counsellors to implement complex interventions based on MI. An attempt to determine the factors that might enable some lay counsellors to adopt MI skills and the Options counselling protocol more readily than others should also be made. In terms of the Options training programme that is to be developed for delivery to counsellors, a more comprehensive module focused specifically on the counselling techniques associated with MI needs to be included. This should be developed with the evaluation tools in mind so that their applicability to evaluating the skill of counsellors is ensured.

\section{Conclusion}

This case study has revealed a number of issues at the organizational and individual levels that need to be addressed in order for the transfer of the Options intervention into the South African ARV treatment programme to be successful. More generally this study has drawn attention to some of the potential barriers to incorporating positive prevention into lowresourced HIV care settings as well as the need to determine the level of expertise required to successfully deliver such complex interventions. Future research should investigate the impact of enhanced training and support on counsellors' ability to implement Options for Health and the skills associated with MI, and should also include a longer term of follow-up in order to allow for (and to determine the impact of) adjustments in response to the problems that are likely to be encountered.

Acknowledgments The authors would like to acknowledge Ms Joanne Croome for providing the booster training session and the evaluation of the transcript, as well as Ms Reshma Ramachandran and Ms Nontobeko Mdudu for their work on data collection. The work of the adherence counsellor in this study is also gratefully acknowledged.

\section{References}

1. UNAIDS. Report on the global AIDS epidemic 2006. Geneva: UNAIDS; 2006.

\section{https://repository.uwc.ac.za/}


2. Western Cape Department of Health. Western Cape antiretroviral monthly summary. 2008.

3. Eisele TP, Mathews C, Chopra M, et al. Changes in risk behaviour among HIV-positive patients during their first year of anti- retroviral therapy in Cape Town, South Africa. AIDS Behav. 2009;13(6):1097-105.

4. Kozal MJ, Amico KR, Chiarella J, et al. Antiretroviral resistance and high-risk transmission behaviour among HIV-positive patients in clinical care. AIDS. 2004;18(11):2185-9.

5. Cornman DH, Kiene SM, Christie S, et al. Clinic-based intervention reduces unprotected sexual behaviour among HIV- infected patients in Kwa-Zulu Natal, South Africa: results of a pilot study. J Acquir Immune Defic Syndr. 2008;48(5):553-60.

6. Schneider H, Hlope H, van Rensburg D. Community health workers and the response to HIV/AIDS in South Africa: tensions and prospects. Health Policy Plan. 2008; April 1-9.

7. Lewin SA, Dick J, Pond P et al. Lay health workers in primary and community health care. Cochrane Database Syst Rev. 2005; (1):CDoo4015.

8. Doherty TM, Coetzee M. Community health workers and professional nurses: defining the roles and understanding the relationships. Public Health Nurs. 2005;22(4):360-5.

9. Mash R, Baldassini G, Mkhatshwa H, Sayeed I, Ndapeua S. Reflections on the training of counsellors in motivational interviewing for programmes for the prevention of mother to child transmission of HIV in sub-Saharan Africa. S Afr Fam Pract. 2008;50(2):53-9.

10. Evangeli M, Engelbrecht S, Swartz L, Turner K, Forsberg L, Soka N. An evaluation of a brief motivational interviewing training course for HIV/AIDS counsellors in Western Cape Province, South Africa. AIDS Care. 2009;21(2):189-96.

11. Moyers TB, Martin T, Manuel JK, Hendrickson SML, Miller WR. Assessing competence in the use of motivational interviewing. J Subst Abuse Treat. 2007;28:19-26.

12. Lane C, Huws-Thomas M, Hood K, Rollnick S, Edwards K, Robling M. Measuring adaptations of motivational interviewing: the development and validation of the behaviour change counselling index (BECCI). Patient Educ Couns. 2005;56(2):166-73. 